\title{
Importance of rings on the microscopic properties of a strong glass
}

\author{
R. Fernández-Perea, F. J. Bermejo, and M. L. Senent \\ Instituto de Estructura de la Materia, Consejo Superior de Investigaciones Científicas, \\ Serrano 123, E-28006 Madrid, Spain
}

(Received 19 March 1996)

\begin{abstract}
An optimized interparticle potential has been developed for its use in computer molecular dynamics simulations of vitreous boron trioxide $\left(v-\mathrm{B}_{2} \mathrm{O}_{3}\right)$ which includes four-particle interactions. This is required to force the formation of planar six-membered (boroxol) rings in such simulations. As a result, a better agreement with the static structure factor is achieved, even though the percentage of atoms taking part in such structures is relatively small. [S0163-1829(96)02934-7]
\end{abstract}

The presence of a substantial amount of regular structures in strong glasses such as vitreous silica or boron trioxide has been a matter of heated debate for a number of years. ${ }^{1}$ The presence of such structures was postulated as a means to explain the origin of sharp peaks in the Raman spectra on the basis of calculations involving a few particles, ${ }^{2}$ or more refined approaches which still involved idealized models of the amorphous solid such as the Bethe lattice. ${ }^{3}$ However, attempts to relate the presence of such structures with discernible features in the diffraction patterns lead to some recent controversies. In fact, results from calculations involving short-range structures only which portray most atoms as being involved in boroxol rings (about eighty percent) are confronted with attempts to reconstruct the structure of the glassy solid by means of Reverse Monte Carlo approaches, ${ }^{4}$ where the best results are obtained if the percentage of boroxol rings is bounded below thirty percent (i.e., even the absence of any of those structures gives a better fit to experiment than those assuming a fraction of fifty percent or above). In fact, attempts to calculate the structure of vitreous $\mathrm{B}_{2} \mathrm{O}_{3}$ using relatively small three-dimensional clusters of atoms forming boroxol rings have often encountered considerable difficulties when trying to reproduce the correct macroscopic density. This contrasts with results from molecular dynamics (MD) simulations ${ }^{5,6}$ which, while satisfactorily accounting for the macroscopic density, have evidenced difficulties in reproducing the presence of a large number of atoms involved in planar, regular rings. On the contrary, such simulation results have shown the presence of cyclic structures albeit severely deformed (i.e., nonplanar) and of varied ring sizes.

A recurrent critique against $\mathrm{MD}$ results on the structure and dynamics of $v-\mathrm{B}_{2} \mathrm{O}_{3}$ has always been the lack of such structures in the computed equilibrium structure ${ }^{2}$ of the glass. Such a shortcoming was rationalized in terms of (a) the huge cooling rates employed in the computer quench from the equilibrium liquid structure or (b) the lack of a more realistic potential function which including four-body interactions stabilizes such planar structures.

Although little can be done to overcome the first of the difficulties referred above since quench rates comparable with experiment would imply vast resources of computer time, we set our endeavors to explore the feasibility of supplementing the potential function described in Refs. 6 and
7 with a four-body term to provide a force which stabilizes regular (i.e., planar) six-membered-ring structures. To start with, several Hartree-Fock calculations on model $\mathrm{B}_{3} \mathrm{O}_{3}(\mathrm{OH})_{3}$ structures were carried out using relatively large basis sets. ${ }^{8}$ After checking that the planar ring configuration of such a cyclic structure represents an absolute minimum in the potential energy surface (i.e., allowing the relaxation of all the relevant degrees of freedom), a set of calculations setting one of the atoms above the plane defined by the rest of five atoms by a fixed distance and allowing for full relaxation of the rest of the degrees of freedom were carried out. The calculations should be considered a bit more sophisticated than the ones reported in Ref. 9 performed using a similar methodology but focused on the estimation of rather different properties (calculation of the frequencies of localized vibrations, nuclear magnetic shielding tensors and nuclear quadrupole resonance parameters).

From such a calculation the explicit functional form for the potential was found to follow

$$
V_{\text {O-B-O-B }}(\tau)=A_{1}[1-\cos (2 \tau)]+A_{2}[1-\cos (8 \tau)] \text {, }
$$

where $\tau$ stands for the dihedral angle subtended by planes O-B-O and B-O-B within a boroxol ring and $A_{i}$ are two constants which had to be rescaled by $1 / 3$ to reproduce the experimental frequencies. The need for such a rescaling has been amply acknowledged even in calculations regarding free-molecule species ${ }^{8}$ so that it did not came as a surprise. On the other hand, the introduction of such a higher-order term made necessary the modification of the two- and threebody potential constants employed in previous calculations, if an acceptable agreement with experimental magnitudes is sought.

To prepare a quench from the stabilized liquid down to the glass, the strategy described in Ref. 7 was followed. That involves equilibration of a liquid configuration at $900 \mathrm{~K}$, where are interactions are assumed to be of two-body type. Once equilibrated, three- and four-body interactions are switched on and allow to reequilibrate for about 100 ps. Cooling down to the glass phase at $300 \mathrm{~K}$ is finally achieved in a simulation lasting 20 ps. Notice that the expression [1] has been parameterized from calculations involving relatively small out-of-plane displacements and consequently its range of validity should be confined to a moderate region of 
TABLE I. Reoptimized values for the potential parameters. Those comprising two-body interactions follow $V_{i j}=A_{i j} \exp \left(-r / \rho_{i j}\right)+\left(z_{i} z_{j} e^{2}\right) / r$ where $\rho_{i j}$ stands for the ionic radius, $z_{i}$ the electronic charge, and $e$ is the fundamental unit of charge. Those for the three-body part follow $V_{i j k}(\theta)=1 / 2 K_{i j k}\left(\theta-\theta_{0}\right)^{2}$ where $\theta_{0}$ stands for the equilibrium bond angle and $K_{i j k}$ its associated force constant, and finally a four-body part is given in terms of the $\tau$ dihedral angle as $V_{\mathrm{O}-\mathrm{B}-\mathrm{O}-\mathrm{B}}(\tau)=A_{1}[1-\cos (2 \tau)]+A_{2}[1-\cos (8 \tau)]$.

\begin{tabular}{|c|c|c|c|c|c|c|c|c|c|}
\hline \multicolumn{3}{|c|}{$\times 10^{3} \mathrm{~kJ} \mathrm{~mol}^{-1}$} & $\rho_{\mathrm{BB}}$ & $\begin{array}{r}\rho_{\mathrm{BO}} \\
\AA\end{array}$ & $\rho_{\mathrm{OO}}$ & $z_{\mathrm{B}}$ & $z_{\mathrm{O}}$ & $\begin{array}{c}K_{\mathrm{O}-\mathrm{B}-\mathrm{O}} \\
\times 10^{3} \mathrm{~kJ}\end{array}$ & $\begin{array}{c}K_{\mathrm{B}-\mathrm{O}-\mathrm{B}} \\
-1 \mathrm{rad}^{-2}\end{array}$ \\
\hline 5.9614 & 33.72 & 75.65 & 0.1 & 0.295 & 0.287 & 2.000 & -1.333 & 1.00 & 0.25 \\
\hline \multicolumn{3}{|c|}{$A_{1} \times 10^{20} \mathrm{~J}$} & \multicolumn{5}{|c|}{3.7606} & $\theta_{0}$ & $\theta_{0}$ \\
\hline \multicolumn{3}{|c|}{$A_{2} \times 10^{20} \mathrm{~J}$} & \multicolumn{5}{|c|}{-0.0712} & 120 & 119 \\
\hline
\end{tabular}

$\tau$ values. In this respect, we have verified that such potential function can safely be used for computations where $\cos (\tau) \geqslant 0.5$, since leaving unbounded such a range it leads to rather severe stabilization problems.

The values for the potential parameters resulting from such a reoptimization are given in Table $\mathrm{I}$.

The calculated distribution of $\tau$ angles for $T=300 \mathrm{~K}$ is shown in Fig. 1, and evidences that most of the angles adopt values close to those allowing partial planarity with a near symmetrical distribution of values for $\tau=0^{\circ}$ and $\tau=180^{\circ}$. As can be seen from such a graph, most of the values for such a geometrical parameter are distributed within an interval of about $20^{\circ}$ about the two most probable values.

As a first check of the quality of the optimized potential, the Fig. 2 shows a comparison of the static structure factors as measured by neutron diffraction, ${ }^{10}$ that calculated using the previous potential ${ }^{6}$ and the present one. As can easily be gauged from the referred graph the calculated $S(Q)$ with either three- or four-body terms reproduce the oscillations above some $4 \AA^{-1}$ in the experimental pattern rather satisfactorily. The present results show a better agreement as far as the shape and height of the first peak in $S(Q)$ is concerned, than the one resulting from the simplified form of the potential. It is worth pointing here that a near perfect match of the experimental first peak can be found at the expense of introducing a severe mismatch with subsequent oscillations, and this is also achievable using the three-body potential only. The results shown in Fig. 2 thus represent a compro-

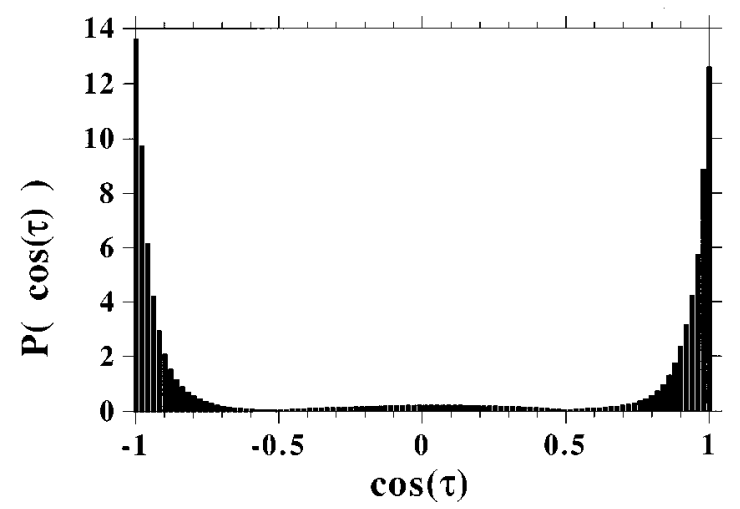

FIG. 1. Computed distribution for dihedral angles. $P[\cos (\tau)]$ stands for the probability that a given dihedral angle has such a value and is given in percentage units. mise between an adequate accounting of the short-range order and an acceptable representation for the first peak. A detailed account of the physical significance of parameters characterizing the first peak has recently been given. ${ }^{11}$ In particular, a comparison between simulation results and experimental data regarding the elastic $S(Q, E=0)$ and static $S(Q)$ quantities was provided for temperatures $5 \mathrm{~K} \leqslant T \leqslant$ $300 \mathrm{~K} .{ }^{11}$ The relevant point to make here regards the somewhat different shape of the first diffraction peak resulting from such an experiment (i.e., employing rather low incident energies) from that observed using total scattering techniques. ${ }^{10}$ Notice that the shape of the peak in the calculated $\mathrm{S}(\mathrm{Q})$ becomes closer to that measured using an incident energy of $5 \mathrm{meV}$ (Ref. 11) than to that of Ref. 10.

As far as the microscopic dynamics is concerned, the main effect of the four-body term can be gauged from inspection of the lower frame of Fig. 2 which displays a comparison between the $Z(E)$ vibrational densities of states as calculated from Fourier transform of the relevant atomic velocity correlation functions for the model which accounts up to three body forces only and the present one. The most noticeable differences between the two generalized frequency distributions concern the appearance of a clear peak centered at $70 \mathrm{meV}$, while a similar feature is seen in the three-body calculation as centered at $80 \mathrm{meV}$, a region where only a shoulder is now seen, as well as the appearance of clear (e.g., well defined) peaks at $90 \mathrm{meV}$ and $100 \mathrm{meV}$. Notice that in the previous calculation, well defined peaks in the $100 \mathrm{meV}$ region were only visible in low temperature runs. ${ }^{6}$ The relevance of such a spectral feature stems from its identification with that arising from the breathing motion of a boroxol ring, ${ }^{2}$ postulated on the basis of the intense, narrow and strongly polarized isolated peak seen at the same frequency in the Raman spectrum. Contrary to what is observed by Raman scattering, the peak observable in inelastic neutron scattering measurements ${ }^{2}$ shows an intensity not too different from those of peaks at $82 \mathrm{meV}$ and $90 \mathrm{meV}$ although its width $[\approx 1.7 \mathrm{meV}$ (Ref. 2)] is about half of that of the other two. A recent inelastic neutron scattering measurement ${ }^{12}$ on the temperature dependence of the intensity of such a peak shows that it develops itself as a well defined feature for temperatures well within the glass phase. Also, its sharpness is a direct consequence of the independence of the peak frequency with the wave number (at least within $4 \AA^{-1} \leqslant Q$ $\leqslant 12 \AA^{-1}$ ). Such an opticlike behavior contrasts however with its extreme sensitivity to alterations in the elastic prop- 

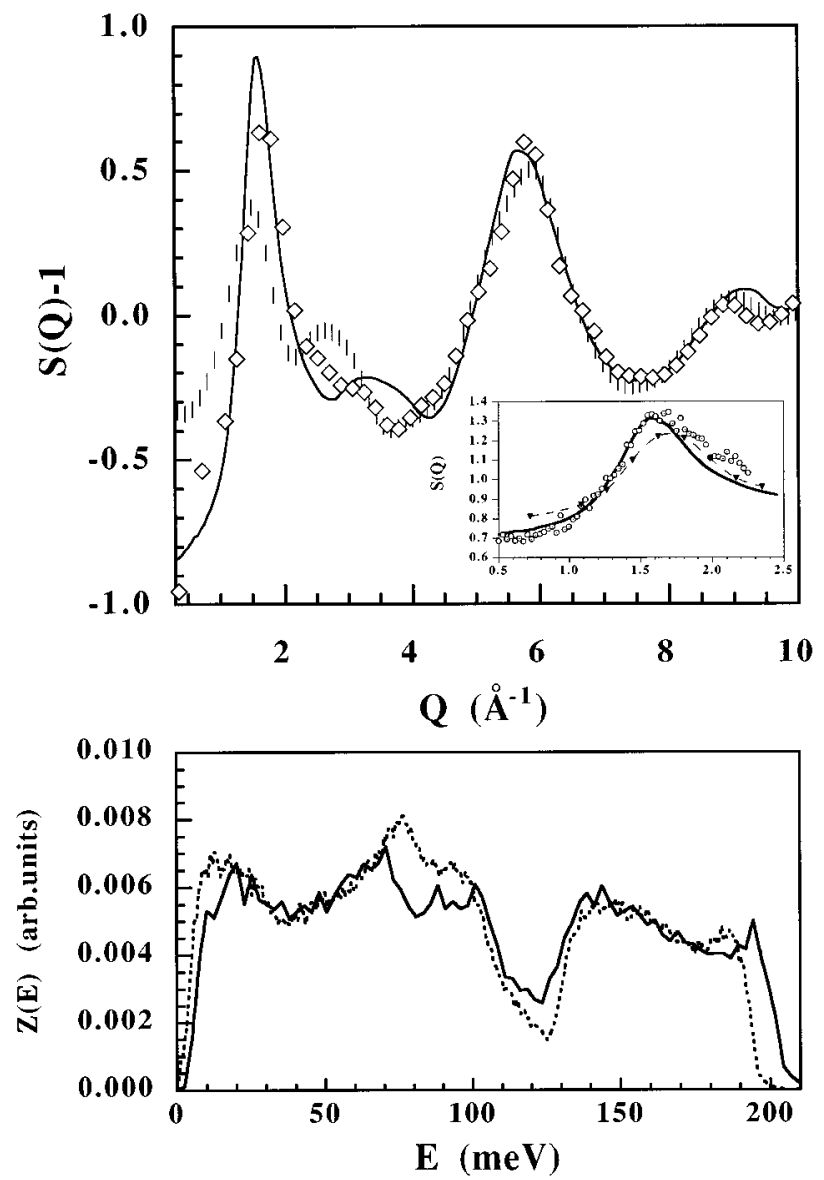

FIG. 2. The upper frame shows a comparison between the experimental diffraction pattern [solid line (Ref. 10)], the one derived from MD computations using a potential including up to three-body terms only (Ref. 6) (vertical bars), and the present results (lozenges). The inset compares two diffraction patterns [solid (Ref. 10), circles with a dot (Ref. 11)] and the present result (dashed line with inverted triangles). The lower frame shows a comparison between the calculated frequency distributions corresponding to the model which includes three-body terms only (dashed line) and the present one (solid line).

erties of the medium, and in fact, both its frequency, intensity and linewidth show a behavior resembling that expected for a mode evidencing a nontrivial (mixed) polarization, ${ }^{17}$ which leads to show kinks in graphs showing the temperature dependence of the peak frequency at temperatures close to the glass transition point $T_{g}=533 \mathrm{~K}$.

An unequivocal assignment of such a peak to the referred mode remains to be produced since most of the work in this direction regards rather simplified models, ${ }^{2,13-15}$ which can be compared with experimental data on semiquantitative grounds at best. Within such context, the present data lend partial support to the idea that four-body forces are involved in the atomic vibrations taking part within such a narrow range of frequencies, since the new calculation shows that a relatively well defined peak, with a width of about $3 \mathrm{meV}$ is now present at $100 \mathrm{meV}$. On the contrary, the assignment of such a peak to a mode involving the six atoms within a boroxol ring seems difficult to be sustained since only two of such rings are present in the 420 atoms (168 B and $252 \mathrm{O}$ ) simulation box.

Forcing the formation of boroxol rings by increasing the strength of the four-body term with respect to the two and three-body ones not only leads to difficulties in equilibration but also produces spurious peaks (spikes) and results in an unphysical redistribution of frequencies in the vibrational density of states but also makes the glass density to go well below the correct value.

In terms of macroscopic thermal properties, the value calculated for the harmonic part of the heat capacity at $\mathrm{T}=300$ $\mathrm{K}$ gives now $13.1 \mathrm{~J} \mathrm{~K}^{-1} \mathrm{~mol}^{-1}$ versus $12.01 \mathrm{~J} \mathrm{~K}^{-1} \mathrm{~mol}^{-1}$ of the calculations involving three-body terms only and compares with the experimental value of $12.31 \mathrm{~J} \mathrm{~K}^{-1} \mathrm{~mol}^{-1}$ for $C_{p}(T=300 \mathrm{~K})$. A quasiharmonic correction is difficult to evaluate because of the paucity of data regarding the temperature dependence of the spectral frequency distributions. An estimate of it can be made on the basis of data regarding the temperature dependence of the frequency of the peak appearing at $\approx 100 \mathrm{meV}$ (Ref. 17) at temperatures within 300-500 K. From there, a value for the derivative $\partial\langle\ln \omega\rangle /\left.\partial T\right|_{P}=-7.2 \times 10^{-4}$ is found, which would represent an increase in heat capacity of some 0.07 per cent in this high temperature limit due to phonon interactions. The volume expansion term $T \gamma_{G} \alpha_{V}$ calculated from expansion data of White et al..$^{16}$ gives some $4.8 \times 10^{-3}$ so that the total quasiharmonic correction would result in a value for the heat capacity smaller than the harmonic one by some 0.4 percent. In consequence the introduction of the four-body term seems to point in the correct direction since leads to a value for the harmonic heat capacity slightly above the experimental one which will be reduced somewhat by the anharmonic interactions.

It seems worth remarking here that the better agreement between experiment and simulation as far as the first peak in $S(Q)$ is concerned, is mainly due to a reoptimization of the values of the (two-body) repulsive cores, playing the fourbody term a rather secondary role.

At any rate, the presence of a narrow peak at $100 \mathrm{meV}$ in the present $Z(E)$, a feature only visible at very low temperatures previously ${ }^{6}$ seems to point to four-body forces as having a dominant role in the vibrational motions leading to such a feature. However, other structures than the motion of a planar six-membered ring may possibly be compatible with such a peak.

Finally, it is worth remarking here that the argument mentioned above regarding the fast thermal quenches performed in simulation work as one of the causes for the absence of boroxol rings in the computations, has, at least to be qualified for the reason that follow. The simulations performed on equilibrated liquid configurations at $T=900 \mathrm{~K}$ show that such structures form within the liquid phase, and the abundance of such rings shows a mild dependence with temperature. In consequence its relative scarcity within the glass does not seem to be ascribable to the rapid quench, since the presence of rings at temperatures not far above from $T_{g}$ will not increase to a large extent.

Work was supported in part by DGICYT (Spain) Grant No. PB92-0114-c03. 
${ }^{1}$ R. Elliot, J. Non-Cryst. Solids 182, 1 (1995) and references therein; see also F.L. Galeener, in Disorder in Condensed Matter Physics, edited by J.A. Blackmann and J. Tagüeña (Oxford Science, Oxford, UK, 1991), p. 45.

${ }^{2}$ A.C. Hannon, D.I. Grimley, R.A. Hulme, A.C. Wright, and R.N. Sinclair, J. Non-Cryst. Solids 177, 299 (1994); A.C. Hannon, A.C. Wright, J.A. Blackman, and R.N. Sinclair, ibid. 182, 78 (1995).

${ }^{3}$ R.A. Barrio, F.L. Castillo-Alvarado, and F.L. Galeener, Phys. Rev. B 44, 7313 (1991).

${ }^{4}$ J. Swenson and L. Börjesson (unpublished).

${ }^{5}$ J.P. Rino, I. Ebbsjö, R.K. Kalia, A. Nakano, and P. Vashista, Phys. Rev. B 47, 3053 (1993).

${ }^{6}$ R. Fernandez-Perea, F.J. Bermejo, and E. Enciso, Phys. Rev. B 53, 6215 (1996).

${ }^{7}$ A.H. Verhoef and H.W. den Hartog, J. Non-Cryst. Solids 146, 267 (1992).

${ }^{8}$ Calculations carried using the Gaussian-92 package using a $6-31 G^{* *}$ basis set. For a review comparing the results of
Hartree-Fock calculations with vibrational frequencies of isolated molecules, see P. Pulay, G. Fogarasi, G. Pongor, J.E. Boggs, and A. Vargha, J. Am. Chem. Soc. 105, 7037 (1983).

${ }^{9}$ J.A. Tosell, J. Non-Cryst. Solids 183, 307 (1995).

${ }^{10}$ P.A.V. Johnson, A.C. Wright, and R.N. Sinclair, J. Non-Cryst. Solids 50, 281 (1982).

${ }^{11}$ F.J. Bermejo, J. Dawidowski, R. Fernández-Perea, and J.L. Martinez, Phys. Rev. B 53, 244 (1996).

${ }^{12}$ A.C. Hannon, F.J. Bermejo, R. Fernández-Perea, R.N. Sinclair, and A.C. Wright (unpublished).

${ }^{13}$ S.J. Williams and S.R. Elliot, Proc. R. Soc. London Ser. A 380, 427 (1982).

${ }^{14}$ Y. Tanaka and M. Katayama, J. Phys. Soc. Jpn. 57, 543 (1988).

${ }^{15}$ F.L. Galeener, Solid State Commun. 44, 1037 (1982).

${ }^{16}$ G.K. White, S.J. Collocot, and J.S. Cook, Phys. Rev. B 29, 4778 (1984).

${ }^{17}$ A.K. Hassan, L.M Torrell, L. Börjesson, and H. Doweidar, Phys. Rev. B 45, 12797 (1992). 